significant number of our patients. The KDIGO guidelines offer a framework to work with our renal colleagues, as many patients will be jointly managed.

References:

[1] Diagnosis, Evaluation, Prevention, and Treatment of Chronic Kidney

[2] Disease-Mineral and Bone Disorder: Synopsis of the Kidney Disease:

[3] Improving Global Outcomes 2017 Clinical Practice Guideline Update. Ann Int Med 2018

Disclosure of Interests: NATALIA CERNOVSCHI: None declared, SHABEENA ZEB: None declared, TRACEY SALTER: None declared, MARK LLOYD Speakers bureau: $£ 700$ into department fund

DOI: 10.1136/annrheumdis-2020-eular.1490

\section{AB0892 1 PREGNANCY AND LACTATION ASSOCIATED OSTEOPOROSIS: FIRST CASE SERIES IN IRAN}

M. Aghaei ${ }^{1}$, S. Sedighi ${ }^{1}$, M. Hassani ${ }^{1}$, M. R. Damirchi ${ }^{1} .{ }^{1}$ Golestan University of Medical Sciences, Golestan Rheumatology Research Center, Gorgan, Iran (Islamic Republic of)

Background: Osteoporosis is a common rheumatologic disorder in postmenopausal women which could lead to morbidities later in life. However, this condition has not been properly studied in premonapausal women.

During pregnancy, the fetus needs a total of 30 grams of calcium for its skeleton and during lactation, $200 \mathrm{mg}$ of calcium is secreted in the breast milk per day which the mother acquires by doubling its intestinal absorption rate. If the calcium intake of the mother is not sufficient to satisfy the fetus' needs, it will be provided by bone resorption, which will decrease the maternal calcium reserves [1].

Pregnancy and Lactation Associated Osteoporosis (PLAO) is a rare condition associated with pregnancy that should be considered in premenopausal women. The most commonly affected sites are the vertebrae and, more rarely, the hips, pubic rami and ribs [2].

An important complication of osteoporosis is fracture and a preemptive diagnosis and treatment thereof, can have drastic effects on the quality of life.

Objectives: Our objective is to document the relevant risk factors, present signs and symptoms, course of illness, and response to treatment in three cases of $\mathrm{PLAO}$. It is quite possible that osteoporosis in pregnancy

and lactation is more frequent than recognized, simply because it is only recognized when an-unexpected fracture occurs[3]. Thus, in this article we are presenting three cases that showcase the need for more rigorous research on PLAO risk factors, the need for screening in high risk patients, and the advantages of early detection in patients' outcome.

Methods: The clinical cases of the patients whose PLAO diagnoses had been confirmed by both a radiologist and a rheumatologist in the past year was extracted. Information related to demographic indices, clinical manifestations, and the treatment methods was evaluated and compared.

Results: In the past year, three patients with a chief complaint of low-back pain have visited our clinic. The first, a 22-year-old woman with a nursing history of 2 months, the next one, a 31-year-old woman with a nursing history of 3 months, and the last, a 22-year-old woman with a nursing history of 4 months. All three patients had low back pain and tenderness. Two out of three patients had deficient vitamin $\mathrm{D}$ levels and the other had a normal one. All three patients had low BMD in lumbar vertebra and MRI imagings indicative of osteoporotic fracture.

Table 1. General and pregnancy-relatedcharacteristics of the case studies

\begin{tabular}{lccc}
\hline Variables & Case 1 & Case 2 & Case 3 \\
\hline Age & 22 & 31 & 22 \\
Nursing duration & 2 months & 3 months & 4 months \\
BMD(lumbar spine) & -3.6 & -3.5 & -3.1 \\
Vitamin D level & $12.6(\mathrm{ng} / \mathrm{ml})$ & $31.6(\mathrm{ng} / \mathrm{ml})$ & $8(\mathrm{ng} / \mathrm{ml})$ \\
Fracture & $\mathrm{T}_{12,} \mathrm{~L}_{1}$ & $\mathrm{~T}_{4,5,6,7,10}, \mathrm{~L}_{2}$ & $\mathrm{~L}_{1,2,3,4,5}$ \\
\hline
\end{tabular}

Conclusion: Since the symptoms of PLAO are often confused with pain in other low-back pain conditions associated with pregnancy, PLAO is a mostly overlooked diagnosis[4]. It is only recognized when an unexpected fracture occurs [3]Therefore, high risk patients with less severe symptoms are usually not diagnosed and thusly, should undergo a proper screening test, so that they are recognized early and the morbid sequelae are averted.

References:

[1] Kovacs, C. S., and S. H. Ralston. "Presentation and Management of Osteoporosis Presenting in Association with Pregnancy or Lactation." Osteoporosis International, vol. 26, no. 9, 2015, pp. 2223-2241., doi:10.1007/ s00198-015-3149-3.
[2] Gregorio, Silvana Di, et al. "Osteoporosis with Vertebral Fractures Associated with Pregnancy and Lactation." Nutrition, vol. 16, no. 11-12, 2000, pp. 1052-1055., doi:10.1016/s0899-9007(00)00430-5.

[3] Bartl, Reiner, and Christoph Bartl. "The Osteoporosis Manual." 2019, doi:10.1007/978-3-030-00731-7.

[4] Eroglu, Semra, et al. "Evaluation of Bone Mineral Density and Its Associated Factors in Postpartum Women." Taiwanese Journal of Obstetrics and Gynecology, vol. 58, no. 6, 2019, pp. 801-804., doi:10.1016/j.tjog.2019.09.013.

Disclosure of Interests: None declared

DOI: 10.1136/annrheumdis-2020-eular.2665

\begin{tabular}{|l|l|}
\hline AB0893 & THE EFFECTS OF THREE DIFFERENT VITAMIN D3 \\
SUPPLEMENTATION REGIMENS IN DEFICIENT \\
SUBJECTS - A RANDOMIZED OPEN-LABEL \\
PARALLEL GROUP STUDY.
\end{tabular}

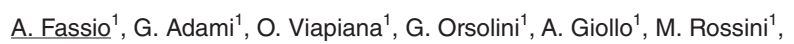
D. Gatti ${ }^{1}{ }^{1}$ Rheumatology Unit, University of Verona, Verona, Italy

Background: Currently, most experts agree that levels of serum $25 \mathrm{OH}$-Vitamin D (25OHD) lower than $20 \mathrm{ng} / \mathrm{mL}$ represent an acceptable threshold for deficiency (1). However, recommendations for vitamin D supplementation vary between scientific societies, and the best regimen to treat deficient patients is still not clear (1)

Objectives: The aim of our study was to compare the pharmacokinetic profile of three different regimes of cholecalciferol supplementation in terms of $250 \mathrm{HD}$ exposure and their safety profiles.

Methods: We evaluated, in healthy subjects affected by vitamin $\mathrm{D}$ deficiency (defined as $250 \mathrm{HD}<20 \mathrm{ng} / \mathrm{mL}$ ), 18 to 60 years of age, the efficacy of three different oral supplementation regimens: daily 10,000lu administered for 8 weeks, weekly 50,000 lu for 12 weeks and biweekly 100,000lu for 12 weeks. Serum 25OHD was dosed at baseline, at week 2, 4, 8 in all three groups and also at week 12 in the 50,000 and 100,000lu groups (the blood sample was taken before the drug administration if scheduled on the same day). Baseline characteristics and $250 \mathrm{HD}$ changes from baseline to the various observation points were tested with ANOVA and t-test. 25OHD was measured by the IDS-ISYSMulti-Discipline automated analyser (Immunodiagnostic System, Boldon, UK) based on chemiluminescence technology. The CV intra-assay measured in our laboratory was 6\% (inter-assay CV $9 \%$ ). The study was authorized by the local ethical committee (protocol DIBA/11. Supported by Abiogen Pharma, Italy).

Results: A total of 75 subjects were randomized to receive one supplementation regimen. The descriptive of the sample at baseline and relative $250 \mathrm{HD}$ levels at the various observation points are reported in table 1. 25OHD increased significantly already at week 2 in all three groups $(p=0.000)$. At week $2,100 \%$ of the subject of the daily regimen group presented $25 \mathrm{OHD}$ levels $>20 \mathrm{ng} / \mathrm{mL}, 96 \%$ in the weekly and $88 \%$ in the biweekly ones.

Table 1. mean values $\pm S D$ at the different observation points.

\begin{tabular}{|c|c|c|c|c|}
\hline Parameter & $\begin{array}{l}\text { Daily } 10.000 \mathrm{Ui} \\
\quad(\mathrm{N}=25)\end{array}$ & $\begin{array}{l}\text { Weekly } 50.000 \\
\text { Ui } \\
\qquad(n=25)\end{array}$ & $\begin{array}{c}\text { Biweekly } \\
100.000 \mathrm{Ui} \\
(\mathrm{N}=25)\end{array}$ & $p$-value \\
\hline M:F & $12: 13$ & $7: 18$ & $12: 13$ & \\
\hline Age (years) & $30.2 \pm 9.9$ & $36.7 \pm 8.7$ & $35.4 \pm 11.0$ & ${ }^{*} 0.059$ \\
\hline Body Weight (kg) & $65.8 \pm 13.2$ & $67.8 \pm 10.8$ & $66.6 \pm 13.7$ & ${ }^{*} \mathrm{NS}$ \\
\hline Height (m) & $1.7 \pm 0.1$ & $1.68 \pm 0.1$ & $1.7 \pm 0.1$ & ${ }^{*} \mathrm{NS}$ \\
\hline BMI & $22.55 \pm 2.7$ & $23.8 \pm 2.2$ & $22.8 \pm 2.7$ & ${ }^{*} \mathrm{NS}$ \\
\hline $\begin{array}{l}\text { Baseline 250HD (ng/ } \\
\mathrm{mL} \text { ) }\end{array}$ & $14.6 \pm 3.9$ & $12.8 \pm 3$ & $13.5 \pm 4.1$ & ${ }^{\star N S}$ \\
\hline 25OHD week 2 & $32.3 \pm 5$ & $26.5 \pm 3.4$ & $25.6 \pm 5$ & ${ }^{\mathrm{a}} 0.007^{\mathrm{b}} 0.000$ \\
\hline 250HD week 4 & $55 \pm 10.1$ & $39.9 \pm 4.2$ & $36.9 \pm 7.2$ & ${ }^{a} 0.000^{b} 0.000$ \\
\hline 25OHD week 8 & $79.1 \pm 16.2$ & $53.5 \pm 7.2$ & $46.4 \pm 8.2$ & $\begin{array}{c}{ }^{\mathrm{a}} 0.000^{\mathrm{b}} 0.000 \\
{ }^{\mathrm{c}} 0.001\end{array}$ \\
\hline 25OHD week 12 & NA & $58.5 \pm 7.8$ & $50.6 \pm 9.6$ & ${ }^{b} 0.000^{c} 0.001$ \\
\hline
\end{tabular}

${ }^{*}$ ANOVA test.

aDaily 10.000 Ui vs weekly $50.000 \mathrm{Ui}$

${ }^{b}$ Daily 10.000 Ui vs Biweekly $100.000 \mathrm{Ui}$

${ }^{c}$ Weekly 50.000 Ui vs biweekly $100.000 \mathrm{Ui}$.

In addition, the $250 \mathrm{H}$ levels of the daily regimen group at week 8 were higher than both the ones of the weekly and the biweekly regimen groups both at week 8 and week 12 ( $p<0.000$ vs both groups).

No serious adverse event occurred.

Conclusion: All the three different regimens proved to be effective in correcting vitamin $D$ deficiency already after 1 months (2 weeks for the daily regimen). $A$ more refracted approach seems to more effective than the bolus-based regimens. The safety profile was excellent in all groups. 
References:

[1] R. Bouillon, Comparative analysis of nutritional guidelines for vitamin D, Nat. Rev. Endocrinol. 13 (2017) 466-479. https://doi.org/10.1038/ nrendo.2017.31.

Disclosure of Interests: Angelo Fassio Speakers bureau: Angelo Fassio reports personal fees from: Abiogen and Novartis, outside the submitted work., Giovanni Adami: None declared, Ombretta Viapiana: None declared, Giovanni Orsolini: None declared, Alessandro Giollo: None declared, Maurizio Rossini Speakers bureau: AbbVie, Abiogen, Amgen, BMS, Eli-Lilly, Novartis, Pfizer, Sanofi, Sandoz and UCB, Davide Gatti Speakers bureau: Davide Gatti reports personal fees from Abiogen, Amgen, Janssen-Cilag, Mundipharma, outside the submitted work.

DOI: 10.1136/annrheumdis-2020-eular.3641

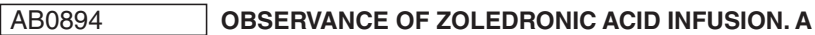 RETROSPECTIVE 3 YEARS STUDY}

\section{J. Zalc ${ }^{1}$, G. Camille ${ }^{1}$, B. Lisa ${ }^{1}$, J. Chloé ${ }^{1}$, B. Frédéric ${ }^{1} .{ }^{1}$ HIA Bégin, Saint Mandé,} France

Background: Osteoporosis is a public health issue. Lack of therapeutic compliance is often a problem in the treatment of osteoroposis, with potentially dramatic consequences. No studies have evaluated the observance of zoledronic acid infusion after 3 years, the time of the therapeutic reassessment.

Objectives: The main objective of assessing the level of compliance was to evaluate the level of zoledronic acid infusion adherence at 1, 2 and 3 year periods, in a cohort of osteoporotic patients on discharge from Begin hospital, following treatment for fracture caused by low-energy trauma. The first infusion was prescribed by rheumatologists, with the following infusions to be prescribed by general practitioners.

Methods: We performed a retrospective observational study initially conducted by written and telephone questionnaires on a population of patients hospitalized in the rheumatology department of HIA Bégin for an osteoporotic fracture. Data was collected between July 2015 and December 2018. A first letter, containing a stamped addressed envelope to the Bégin hospital for ease of reply, was sent to the patients selected for the study. The protocol had to be modified following a very low response rate, unaided by bad quality addresses. We then tried to contact the patients by phone 3 times and, if unable to reach them, we called their general practitioners on 3 occasions.

Results: 94 patients were initially selected. Every year, we retained within the study patients who had followed their annual zoledronic acid infusion protocol. Taking into account all 94 patients, adherence level for the first infusion was $41.4 \%$, down to $29.7 \%$ for the second infusion and down to $12.8 \%$ for the third infusion.

For those who had the first infusion performed, adherence level for the second infusion was $71.8 \%$, down to $30.8 \%$ for the third infusion.

Conclusion: The observance and follow-up of zoledronic acid infusion in France by general practitioners is not adequate. Follow-up measures on an annual basis by the rheumatologist could significantly improve adherence.

References:

[1] Curtis JR et al. Adherence with intravenous zoledronate and intravenous ibandronate in the United States Medicare population. Arthritis care \& research. 2012;64:1054-60.

[2] Jaleel $A$ et al. Improving drug adherence in osteoporosis: an update on more recent studies. Therapeutic advances in musculoskeletal disease. 2018;10:141-9.

Acknowledgments: None

Disclosure of Interests: None declared

DOI: 10.1136/annrheumdis-2020-eular.4841

\section{AB0895 RELATIONSHIP BETWEEN BONE MINERAL DENSITY, INFLAMMATORY ACTIVITY AND AUTOIMMUNITY IN A COHORT OF EARLY RHEUMATOID ARTHRITIS PATIENTS}

S. Garcia Carazo ${ }^{1}$, D. Peiteado2, A. Villalva ${ }^{1}$, L. Nuño ${ }^{1}$, M. Diaz ${ }^{3}$, A. Balsa ${ }^{1}$, P. Aguado ${ }^{1} .{ }^{1}$ Hospital La Paz, Rheumatology, Madrid, Spain; ${ }^{1}$ Hospital La Paz, Rheumatology, Madrid, Spain; ${ }^{3}$ Hospital La Paz, Biostatistics, Madrid, Spain

Background: The etiology of bone loss in Rheumatoid Arthritis (RA) is multifactorial and systemic inflammation plays a relevant role. Recently, a relationship between autoimmunity and bone mineral density (BMD) has been described in patients with RA.
Objectives: To study BMD and biochemical parameters of bone metabolism in a cohort of patients with early rheumatoid arthritis, and assess the relationship between them and autoimmunity and other markers of inflammation.

Methods: A prospective longitudinal study was performed. 128 patients from an early Rheumatoid Arthritis Unit (ERAU) were included. All of them fullfilled ACR 2010 classification criteria for RA. Demographic, clinical, biochemical, immunological, radiological and densitometric data, and also inflammatory activity index DAS 28, HAQ functional index, were collected. Any value $>20 \mathrm{IU} / \mathrm{mL}$ for RF and $>30 \mathrm{IU} / \mathrm{mL}$ for ACPA was defined as positive.

Results: Between January 2009 and June 2017, 801 patients were evaluated in our ERAU. After two years of follow-up, the most frequent definitive diagnoses were: Early RA 221 (27.6\%), Undifferentiated Arthritis 97 (12.1\%), Psoriatic Arthritis 62 (7.7\%), Spondyloarthritis 54 (6.7\%) and autoimmune Diseases $28(3.4 \%)$.

From the 128 patients with early rheumatoid arthritis evaluated, 104 (81.9\%) were ACPA positive and $98(77.2 \%)$ FR positive. The mean BMD in the total column was $0.96 \pm 0.14 \mathrm{~g} / \mathrm{cm} 2$ and in the femoral neck was $0.76 \pm 0.12 \mathrm{~g} / \mathrm{cm} 2$. No correlation of BMD with autoimmunity markers was found in either of the two locations studied, while a negative relationship between BMD and the PCR inflammation marker (BMD femoral neck: rho-0.203, $p=0.027$ and BMD lumbar spine rho $=-0.27$, p 0.003) was found. The BMD did not correlate with DAS28 nor the HAQ index.

The mean baseline serum calcidiol value was $20.7 \pm 8 \mathrm{ng} / \mathrm{ml}$, and a negative correlation of basal serum calcidiol with the functional $\mathrm{HAQ}$ index was observed ( $r h o=-0.23, p=0.008$ ). No correlation between other autoimmunity (FR and ACPA) and inflammation (VSG, PCR and DAS 28) markers and vitamin $D$ was found.

Conclusion: The BMD in patients with early rheumatoid arthritis of our cohort correlates with the PCR inflammation marker. Unlike other studies shows, in our cohort, serological autoimmunity factors do not have shown to have an independent effect on BMD.

\section{References:}

[1] Llorente I, Merino M, Ortiz AM, et al. Anti-citrullinated protein antibodies are associated with decreased bone mineral density: baseline data from a register of early arthritis patients. Rheumatol Int (2017) 37:799-806

Disclosure of Interests: Sara Garcia Carazo: None declared, Diana Peiteado: None declared, Alejandro Villalva: None declared, Laura Nuño: None declared Mariana Diaz: None declared, Alejandro Balsa Grant/research support from: BMS, Roche, Consultant of: AbbVie, Gilead, Lilly, Pfizer, UCB, Sanofi, Sandoz, Speakers bureau: AbbVie, Lilly, Sanofi, Novartis, Pfizer, UCB, Roche, Nordic Sandoz, Pilar Aguado: None declared DOI: 10.1136/annrheumdis-2020-eular.6267

\section{AB0896 \\ EFFECTIVENESS OF SACROPLASTY IN THE MANAGEMENT OF OSTEOPOROTIC SACRAL FRACTURE IN ELDERLY PATIENTS}

S. Hecquet ${ }^{1}$, F. Verhoeven ${ }^{1}$, S. Aubry ${ }^{2}$, C. Prati ${ }^{1}$, D. Wendling ${ }^{1} .{ }^{1} \mathrm{CHRU}$ Jean Minjoz, Rheumatology, Besançon, France; ${ }^{2} \mathrm{CHRU}$ Jean Minjoz, Radiology, Besançon, France

Background: Sacral fractures are a source of pain leading to loss of autonomy in elderly patients. Sacroplasty may be an effective alternative of conservative medical treatment.

Objectives: To evaluate the short-term analgesic effect of sacroplasty compared to conservative treatment in patients with osteoporotic sacral fractures

Methods: This is a retrospective study of cases of osteoporotic sacral fractures treated with sacroplasty, compared with cases treated with conservative medical procedure over the same period. Outcome was evaluated by pain (Visual analogic scale) short-term (one month) evolution and side effects occurrence.

Results: From January 2009 to June 2019, eleven patients were treated with sacroplasty for osteoporotic fractures at the Besançon University Hospital Centre. These were compared to 12 patients with osteoporotic sacral fracture with exclusive medical management, as a control group. The two groups were similar in age, gender and pain level at baseline. The median VAS was 7/10 in both groups at baseline, In the sacroplasty group, a significant decrease of pain was observed over the two first weeks, with a tendency remaining at day 30 . There were no significant differences in the conservative treatment group at one week $(p=0.2)$, fourteen days $(p=0.6)$ and thirty days $(p=0.7)$ compared to basa assessment.

When comparing the sacroplasty group and the conservative treatment group, no differences were noted at baseline between the two groups, there was a significant difference between the two groups the following day $(p=0.001)$, one 